No. $2004-53$

OBLIGATION RULES FOR MINIMUM COST SPANNING TREE SITUATIONS AND THEIR MONOTONICITY PROPERTIES

By S.H. Tijs, R. Branzei, S. Moretti, H.W. Norde

June 2004

ISSN 0924-7815

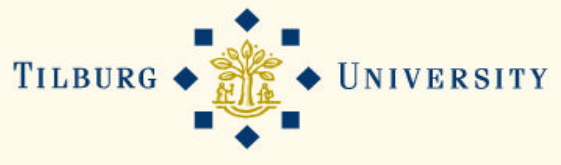




\title{
Obligation rules for minimum cost spanning tree situations and their monotonicity properties.
}

\author{
Stef Tijs ${ }^{1}$, Rodica Branzei ${ }^{2}$, Stefano Moretti ${ }^{3}$, Henk Norde ${ }^{4}$
}

June 9, 2004

Abstract: We introduce the class of Obligation rules for minimum cost spanning tree situations. The main result of this paper is that such rules are cost monotonic and induce also population monotonic allocation schemes. Another characteristic of Obligation rules is that they assign to a minimum cost spanning tree situation a vector of cost contributions which can be obtained as product of a double stochastic matrix with the cost vector of edges in the optimal tree provided by the Kruskal algorithm. It turns out that the Potters value ( $P$-value) is an element of this class.

Key-words: minimum cost spanning tree games, cost monotonicity, population monotonic allocation schemes.

\section{Introduction}

A connection problem arises in the presence of a group of agents, each of which needs to be connected directly or via other agents to a source. If connections among agents are costly, then each agent will evaluate the opportunity of cooperating with other agents in order to reduce costs. In fact, if a group of agents decides to cooperate, a configuration of links which minimizes the total cost of connection is provided by a minimum cost spanning tree.

\footnotetext{
${ }^{1}$ Department of Mathematics, University of Genoa, Italy and CentER and Department of Econometrics and Operations Research, Tilburg University, The Netherlands.

${ }^{2}$ Faculty of Computer Science, "Alexandru Ioan Cuza" University, Iasi, Romania.

${ }^{3}$ Department of Mathematics, University of Genoa and Department of Environmental Epidemiology, National Cancer Research Institute of Genoa, Italy.

${ }^{4}$ CentER and Department of Econometrics and Operations Research, Tilburg University, The Netherlands.
} 
However, solving the cost-minimization problem is only part of the problem: agents must still support the cost of the minimum cost spanning tree and then a cost allocation problem has to be addressed.

This class of allocation problems has been tackled with the aid of cooperative game theory since the basic paper of Bird (1976). For a detailed discussion of the problem let us refer to the dissertations of Aarts (1994) and Feltkamp (1995), and to the papers of Granot and Huberman (1981).

Many cost allocation methods have been proposed and, as usual, different properties have been considered as well, also in view of the applied economic framework. In many applications the cardinality of the set of agents can vary in time, and also increasing or decreasing of connection costs may occur.

Consider, for instance, a wireless telecommunication network where agents are operators of transmitters for traffic exchange and the source is the central hub station. Agents can decide to communicate directly with the main exchange hub, by means of powerful and very expensive transmitters, or, alternatively, to cooperate and construct a wireless network of less powerful, and consequently, cheaper transmitters. Since transmissions are costly, such a situation can be handled as a minimum cost spanning tree problem. Moreover, new owners of transmitters can be willing to enter the network and the cost of connection can increase (i.e. to improve quality and quantity of services supplied) or decrease (i.e. by improving telecommunication technologies). Of course, in all the connection situations suitable to evolve with time, stability conditions satisfied for the original situation cannot guarantee cooperation among agents also under the new conditions. Therefore many authors have focused their attention in finding allocation methods which can keep, in the most general setting, incentives for cooperation also under modifications in the population of agents and in the structure of connection costs.

In the papers of Kent and Skorin-Kapov (1996), Moretti et al. (2002), and Norde et al. (2004), the question of the existence of population monotonic allocation schemes (pmas) (Sprumont (1990)) is central. A pmas provides a cost allocation vector for every coalition in a monotonic way, i.e. the cost allocated to some player does not increase if the coalition to which he belongs becomes larger.

In the paper of Dutta and Kar (2002), cost monotonic allocation rules have been studied, requiring that the cost allocated to agent $i$ does not increase if the cost of a link involving $i$ goes down, nothing else changing in the network.

In this paper, we introduce a class of allocation rules for minimum cost spanning situations, namely the class of Obligation rules, and show that they have nice monotonicity properties: cost monotonicity and population 
monotonicity. Actually, our concept is stronger than the concept of cost monotonicity introduced in Dutta and Kar (2002). We simply impose that if some connection costs go down, then no agents will pay more.

It turns out that particular rules in this class are the $P$-value (Branzei et al. (2003)) and the $P^{\tau}$-values, for each ordering $\tau$ of the players (Norde et al. (2004)). Moreover it is shown that the $P$-value is the average of the $P^{\tau}$-values over all the possible orderings $\tau$.

We start with some preliminaries in the next section. In section 3 Obligation rules are introduced starting from the general notion of obligation maps, and some basic properties are studied. In section 4 it is shown that Obligation rules are cost monotonic and induce population monotonic allocation schemes.

\section{Preliminaries and notations}

An (undirected) graph is a pair $\langle V, E\rangle$, where $V$ is a set of vertices or nodes and $E$ is a set of edges $e$ of the form $\{i, j\}$ with $i, j \in V, i \neq j$. The complete graph on a set $V$ of vertices is the graph $\left\langle V, E_{V}\right\rangle$, where $E_{V}=\{\{i, j\} \mid i, j \in V$ and $i \neq j\}$. A path between $i$ and $j$ in a graph $\langle V, E>$ is a sequence of nodes $i=i_{0}, i_{1}, \ldots, i_{k}=j, k \geq 1$, such that $\left\{i_{s}, i_{s+1}\right\} \in E$ for each $s \in\{0, \ldots, k-1\}$. A cycle in $\langle V, E\rangle$ is a path from $i$ to $i$ for some $i \in V$. Two nodes $i, j \in V$ are connected in $\langle V, E\rangle$ if $i=j$ or if there exists a path between $i$ and $j$ in $E$. A connected component of $V$ in $<V, E>$ is a maximal subset of $V$ with the property that any two nodes in this subset are connected in $\langle V, E\rangle$.

Now, we consider minimum cost spanning tree (mcst) situations. In a mcst situation a set $N=\{1, \ldots, n\}$ of agents is involved willing to be connected as cheap as possible to a source (i.e. a supplier of a service) denoted by 0 . In the sequel we use the notation $N^{\prime}=N \cup\{0\}$. An mcst situation can be represented by a tuple $\left\langle N^{\prime}, E_{N^{\prime}}, w\right\rangle$, where $\left\langle N^{\prime}, E_{N^{\prime}}\right\rangle$ is the complete graph on the set $N^{\prime}$ of nodes or vertices, and $w: E_{N^{\prime}} \rightarrow \mathbb{R}_{+}$is a map which assigns to each edge $e \in E_{N^{\prime}}$ a nonnegative number $w(e)$ representing the weight or cost of edge $e$. We call $w$ a weight function. Since in our paper the graph of possible edges is always the complete graph, we simply denote an mcst situation with set of users $N$, source 0 , and weight function $w$ by $\left.<N^{\prime}, w\right\rangle$. Often we identify an mest situation $\left\langle N^{\prime}, w\right\rangle$ with the corresponding weight function $w$. We denote by $\mathcal{W}^{N^{\prime}}$ the set of all mcst situations $<N^{\prime}, w>($ or $w)$ with node set $N^{\prime}$. For each $S \subseteq N$, one can consider the mcst subsituation $\left\langle S^{\prime}, w_{\mid S^{\prime}}\right\rangle$, where $S^{\prime}=S \cup\{0\}$ and $w_{\mid S^{\prime}}: E_{S^{\prime}} \rightarrow R_{+}$is the restriction of the weight function $w$ to $E_{S^{\prime}} \subseteq E_{N^{\prime}}$, i.e. $w_{\mid S^{\prime}}(e)=w(e)$ for 
each $e \in E_{S^{\prime}}$.

Let $\left\langle N^{\prime}, w\right\rangle$ be an mest situation. Two nodes $i$ and $j$ are called $\left(w, N^{\prime}\right)$ connected if $i=j$ or if there exists a sequence of nodes $i=i_{0}, \ldots, i_{k}=j$ in $N^{\prime}, k \geq 1$, with $w\left(\left\{i_{s}, i_{s+1}\right\}\right)=0$ for every $s \in\{0, \ldots, k-1\}$.

We define the set $\Sigma_{E_{N^{\prime}}}$ of linear orders on $E_{N^{\prime}}$ as the set of all bijections $\sigma:\left\{1, \ldots,\left|E_{N^{\prime}}\right|\right\} \rightarrow E_{N^{\prime}}$, where $\left|E_{N^{\prime}}\right|$ is the cardinality of the set $E_{N^{\prime}}$. For each mcst situation $\left\langle N^{\prime}, w>\right.$ there exists at least one linear order $\sigma \in \Sigma_{E_{N^{\prime}}}$ such that $w(\sigma(1)) \leq w(\sigma(2)) \leq \ldots \leq w\left(\sigma\left(\left|E_{N^{\prime}}\right|\right)\right)$. We denote by $w^{\sigma}$ the column vector $\left(w(\sigma(1)), w(\sigma(2)), \ldots, w\left(\sigma\left(\left|E_{N^{\prime}}\right|\right)\right)\right)^{t}$.

For any $\sigma \in \Sigma_{E_{N^{\prime}}}$ we define the set

$$
K^{\sigma}=\left\{w \in \mathbb{R}_{+}^{E_{N^{\prime}}} \mid w(\sigma(1)) \leq w(\sigma(2)) \leq \ldots \leq w\left(\sigma\left(\left|E_{N^{\prime}}\right|\right)\right)\right\}
$$

Any mcst situation gives rise to two problems: the construction of a network $\Gamma \subseteq E_{N^{\prime}}$ of minimal cost connecting all users to the source, and a cost sharing problem of distributing this cost in a fair way among users. The cost of a network $\Gamma$ is $w(\Gamma)=\sum_{e \in \Gamma} w(e)$. A network $\Gamma$ is a spanning network on $S^{\prime} \subseteq N^{\prime}$ if for every $e \in \Gamma$ we have $e \in E_{S^{\prime}}$ and for every $i \in S$ there is a path in $\Gamma$ from $i$ to the source. To construct a minimum cost spanning network $\Gamma$ on $N^{\prime}$ we use in this paper the Kruskal algorithm (Kruskal (1956)), where the edges are considered one by one according to non-decreasing cost, and an edge is either rejected, if it generates a cycle with the edges already constructed, or it is constructed, otherwise.

Let $\left\langle N^{\prime}, w\right\rangle$ be an mcst situation. The minimum cost spanning tree game $\left(N, c_{w}\right)$ (or simply $c_{w}$ ), corresponding to $\left\langle N^{\prime}, w\right\rangle$, is defined by

$$
c_{w}(S)=\min \left\{w(\Gamma) \mid \Gamma \text { is a spanning network on } S^{\prime}\right\}
$$

for every $S \in 2^{N} \backslash\{\emptyset\}$, where $2^{N}$ stands for the power set of the player set $N$, with the convention that $c_{w}(\emptyset)=0$.

We call a map $F: \mathcal{W}^{N^{\prime}} \rightarrow \mathbb{R}^{N}$ assigning to every mcst situation $w$ a unique cost allocation in $\mathbb{R}^{N}$ a solution. A solution $F$ is efficient if we have $\sum_{i \in N} F_{i}(w)=w(\Gamma)$ for each $w \in \mathcal{W}^{N^{\prime}}$, where $\Gamma$ is a spanning network on $N^{\prime}$ of minimal cost. A solution $F$ has the carrier property if $F_{i}(w)=0$ for each $w \in \mathcal{W}^{N^{\prime}}$ and for each $i \in N$ such that $i$ is $\left(w, N^{\prime}\right)$-connected to 0 .

Finally a population monotonic allocation scheme or pmas (Sprumont (1990)) for the game $(N, c)$ is a table $x=\left\{x_{S, i}\right\}_{S \in 2^{N} \backslash\{\emptyset\}, i \in S}$ with the properties

i) $\sum_{i \in S} x_{S, i}=c(S)$ for all $S \in 2^{N} \backslash\{\emptyset\}$;

ii) $x_{S, i} \geq x_{T, i}$ for all $S, T \in 2^{N} \backslash\{\emptyset\}$ and $i \in N$ with $i \in S \subset T$. 
Note that conditions (i) and (ii) imply that each row of this table is a core element of the corresponding subgame of the game $(N, c)$ (cf. Sprumont (1990)).

\section{Obligation rules}

Let $\Delta(N)=\left\{x \in \mathbb{R}_{+}^{N} \mid \sum_{i \in N} x_{i}=1\right\}$. The sub-simplex $\Delta(S)$ of $\Delta(N)$ given by $\Delta(S)=\left\{x \in \Delta(N) \mid \sum_{i \in S} x_{i}=1\right\}$ is called, for reasons to be clarified later, the set of obligation vectors of $S$. An obligation function is a map $o: 2^{N} \backslash\{\emptyset\} \rightarrow \Delta(N)$ assigning to each $S \in 2^{N} \backslash\{\emptyset\}$ an obligation vector $o(S) \in \Delta(S)$ in such a way that for each $S, T \in 2^{N} \backslash\{\emptyset\}$ with $S \subset T$ and for each $i \in S$

$$
o_{i}(S) \geq o_{i}(T)
$$

Such an obligation function $o$ on $2^{N} \backslash\{\emptyset\}$ induces an obligation map $\hat{o}$ : $\Theta(N \cup\{0\}) \rightarrow \mathbb{R}^{N}$, where $\Theta(N \cup\{0\})$ is the family of partitions of $N \cup\{0\}$, and $\hat{o}(\theta)=\sum_{S \in \theta, 0 \notin S} o(S)$ for each $\theta \in \Theta(N \cup\{0\})$.

If $\theta=\{N \cup\{0\}\}$, then the resulting empty sum is assumed, by definition, to be the $|N|$-vector of zeroes: $\hat{o}(\theta)=0 \in \mathbb{R}^{N}$.

Example 1 Let $o^{*}: 2^{N} \backslash\{\emptyset\} \rightarrow \Delta(N)$ be defined by $o^{*}(S)=\frac{e^{S}}{|S|}$ for each $S \in 2^{N} \backslash\{\emptyset\}$, where $e^{S}$ is the $|N|$-vector such that $e_{i}^{S}=1$ if $i \in S$ and $e_{i}^{S}=0$ if $i \in N \backslash S$. Then $o^{*}$ is an obligation function and the corresponding obligation map is

$$
\hat{o}_{i}^{*}(\theta)= \begin{cases}|S(\theta, i)|^{-1} & \text { if } 0 \notin S(\theta, i) \\ 0 & \text { otherwise, }\end{cases}
$$

for each $\theta \in \Theta(N \cup\{0\})$ and each $i \in N$. Here $S(\theta, i) \in \theta$ is the set to which $i$ belongs.

Note that $o^{*}(S)$ is the barycenter of $\Delta(S)$ and for $N=\{1,2,3,4\}, \theta=$ $\{\{1,2\},\{0,3\},\{4\}\}$ we have $o^{*}(\theta)=\left(\frac{1}{2}, \frac{1}{2}, 0,1\right)$.

Example 2 Given a bijection $\tau: N \rightarrow\{1,2, \ldots,|N|\}$, let $o^{\tau}$ on $2^{N} \backslash\{\emptyset\}$ be the obligation function such that for each $S \in 2^{N} \backslash\{\emptyset\}$ and $i \in N$

$$
o_{i}^{\tau}(S)= \begin{cases}1 & \text { if } \tau(i)=\min \{\tau(k) \mid k \in S\} \\ 0 & \text { otherwise. }\end{cases}
$$

If $N=\{1,2,3,4\}, \theta=\{\{1,2\},\{0,3\},\{4\}\}, \tau(1)=4, \tau(2)=3, \tau(3)=1$ and $\tau(4)=2$, then $\hat{o}^{\tau}(\theta)=o^{\tau}(\{1,2\})+o^{\tau}(\{4\})=(0,1,0,1)$. 
Example 3 Let $\nu \in \mathbb{R}_{++}^{N}$ be a vector of strictly positive real values. Let $o^{\nu}: 2^{N} \backslash\{\emptyset\} \rightarrow \Delta(N)$ be defined by

$$
o_{i}^{\nu}(S)= \begin{cases}\frac{\nu_{i}}{\sum_{j \in S} \nu_{j}} & \text { if } i \in S \\ 0 & \text { otherwise. }\end{cases}
$$

Then $o^{\nu}$ is an obligation function. Note that if $\nu_{i}=1$ for each $i \in N$, then $o_{i}^{\nu}(S)=o_{i}^{*}(S)$ for each $S \in 2^{N} \backslash\{\emptyset\}$, where $o^{*}(S)$ is as in Example 1.

Example 4 Let $U \subset N$ and let $o^{U}: 2^{N} \backslash\{\emptyset\} \rightarrow \Delta(N)$ be such that

$$
o_{i}^{U}(S)= \begin{cases}|S|^{-1} & \text { if } i \in S \text { and } U \nsubseteq S \\ |U|^{-1} & \text { if } i \in U \text { and } U \subseteq S \\ 0 & \text { otherwise. }\end{cases}
$$

Then, if $N=\{1,2,3,4\}$ and $U=\{2,3\}, o^{U}(\{1,2,4\})=\left(\frac{1}{3}, \frac{1}{3}, 0, \frac{1}{3}\right)$ and $o^{U}(\{1,2,3,4\})=\left(0, \frac{1}{2}, \frac{1}{2}, 0\right)$. So $o^{U}$ is not an obligation function since it does not satisfy condition (1).

Remark 1 Let $o^{\bullet}, o^{\circ}: 2^{N} \backslash\{\emptyset\} \rightarrow \Delta(N)$ be two distinct obligation functions. For each $\alpha \in[0,1]$ let $o^{\alpha}: 2^{N} \backslash\{\emptyset\} \rightarrow \Delta(N)$ be defined by $o^{\alpha}(S)=\alpha o^{\bullet}(S)+$ $(1-\alpha) o^{\circ}(S)$ for each $S \in 2^{N} \backslash\{\emptyset\}$. Then $\sum_{i \in S} o_{i}^{\alpha}(S)=\sum_{i \in S}\left(\alpha o_{i}^{\bullet}(S)+\right.$ $\left.(1-\alpha) o_{i}^{\circ}(S)\right)=1$. Moreover, since condition (1) holds both for $o^{\bullet}$ and $o^{\circ}$, condition (1) holds for their convex combination $o^{\alpha}$ too. Therefore, $o^{\alpha}$ is an obligation function which induces the corresponding obligation map $\hat{o}^{\alpha}(\theta)=$ $\alpha \hat{o}^{\bullet}(\theta)+(1-\alpha) \hat{o}^{\circ}(\theta)$ for each $\theta \in \Theta(N \cup\{0\})$.

Let $w \in \mathcal{W}^{N^{\prime}}$ and let $\sigma \in \Sigma_{E_{N^{\prime}}}$ be such that $w \in K^{\sigma}$. We can consider a sequence of precisely $\left|E_{N^{\prime}}\right|+1$ graphs $<N^{\prime}, F^{\sigma, 0}>,<N^{\prime}, F^{\sigma, 1}>, \ldots$, $<N^{\prime}, F^{\sigma,\left|E_{N^{\prime}}\right|}>$ such that $F^{\sigma, 0}=\emptyset, F^{\sigma, k}=F^{\sigma, k-1} \cup\{\sigma(k)\}$ for each $k \in\left\{1, \ldots,\left|E_{N^{\prime}}\right|\right\}$. For each graph $<N^{\prime}, F^{\sigma, k}>$, with $k \in\left\{0,1, \ldots,\left|E_{N^{\prime}}\right|\right\}$, let $\pi^{\sigma, k}$ be the partition of $N \cup\{0\}$ consisting of the connected components of $N^{\prime}$ in $\left\langle N^{\prime}, F^{\sigma, k}>\right.$.

Remark 2 Note that for each $k \in\left\{1, \ldots,\left|E_{N^{\prime}}\right|\right\}, \pi^{\sigma, k}$ is either equal to $\pi^{\sigma, k-1}$ or is obtained from $\pi^{\sigma, k-1}$ by forming the union of two elements of $\pi^{\sigma, k-1}$.

Now we define recursively a function $\rho^{\sigma}:\{0,1, \ldots,|N|\} \rightarrow\left\{0,1, \ldots,\left|E_{N^{\prime}}\right|\right\}$ by

$$
\text { - } \rho^{\sigma}(0)=0
$$


- $\rho^{\sigma}(j)=\min \left\{k \in\left\{\rho^{\sigma}(j-1)+1, \ldots,\left|E_{N^{\prime}}\right|\right\} \mid \pi^{\sigma, k} \neq \pi^{\sigma, \rho^{\sigma}(j-1)}\right\}$

for each $j \in\{1, \ldots,|N|\}$.

Note that $\pi^{\sigma, \rho^{\sigma}(i)} \neq \pi^{\sigma, \rho^{\sigma}(j)}$ for each $i, j \in\{0,1, \ldots,|N|\}$ with $i \neq j$, and $\sigma\left(\rho^{\sigma}(1)\right), \ldots, \sigma\left(\rho^{\sigma}(|N|)\right)$ correspond to the $|N|$ accepted edges in the Kruskal procedure based on the ordering $\sigma$.

Example 5 Consider the mcst situation $\left\langle N^{\prime}, w\right\rangle$ with $N^{\prime}=\{0,1,2,3\}$ and $w$ as depicted in Figure 1. Note that $w \in K^{\sigma}$, with $\sigma(1)=\{1,3\}$, $\sigma(2)=\{1,2\}, \sigma(3)=\{2,3\}, \sigma(4)=\{1,0\}, \sigma(5)=\{2,0\}, \sigma(6)=\{3,0\}$.

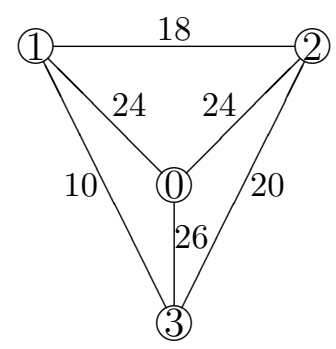

Figure 1: An mcst situation with three agents.

The sequence of seven graphs $\left\langle N^{\prime}, F^{\sigma, k}\right\rangle$ and the corresponding sequence of partitions $\pi^{\sigma, k}$ are shown in the following table

\begin{tabular}{l|l|l}
$k$ & $F^{\sigma, k}$ & $\pi^{\sigma, k}$ \\
\hline & & \\
0 & $\{\emptyset\}$ & $\{\{0\},\{1\},\{2\},\{3\}\}$ \\
1 & $\{\{1,3\}\}$ & $\{\{0\},\{1,3\},\{2\}\}$ \\
2 & $\{\{1,3\},\{1,2\}\}$ & $\{\{0\},\{1,2,3\}\}$ \\
3 & $\{\{1,3\},\{1,2\},\{2,3\}\}$ & $\{\{0\},\{1,2,3\}\}$ \\
4 & $\{\{1,3\},\{1,2\},\{2,3\},\{1,0\}\}$ & $\{N \cup\{0\}\}$ \\
5 & $\{\{1,3\},\{1,2\},\{2,3\},\{1,0\},\{2,0\}\}$ & $\{N \cup\{0\}\}$ \\
6 & $\{\{1,3\},\{1,2\},\{2,3\},\{1,0\},\{2,0\},\{3,0\}\}$ & $\{N \cup\{0\}\}$
\end{tabular}

Then $\rho^{\sigma}(0)=0, \rho^{\sigma}(1)=1, \rho^{\sigma}(2)=2, \rho^{\sigma}(3)=4$.

Definition 1 Let $\hat{o}$ be an obligation map on $\Theta(N \cup\{0\})$. Let $\sigma \in \Sigma_{E_{N^{\prime}}}$. The contribution matrix w.r.t $\hat{o}$ and $\sigma$ is the matrix $D^{\sigma, \hat{o}} \in \mathbb{R}^{N \times E_{N^{\prime}}}$ where the rows correspond to the agents and the columns to the edges, and where

$$
D_{i k}^{\sigma, \hat{o}}=\hat{o}_{i}\left(\pi^{\sigma, k-1}\right)-\hat{o}_{i}\left(\pi^{\sigma, k}\right)
$$

for each $i \in N$ and each $k \in\left\{1, \ldots,\left|E_{N^{\prime}}\right|\right\}$. 
Some characteristics of the contribution matrix are given in the following proposition.

Proposition 1 Let $\hat{o}$ be an obligation map on $\Theta(N \cup\{0\})$. Let $\sigma \in \Sigma_{E_{N^{\prime}}}$. Then $D^{\sigma, \hat{o}}$ is a nonnegative matrix for which each row sum is equal to 1 and the $\rho^{\sigma}(j)$-th column sum is equal to 1 for each $j \in\{1, \ldots,|N|\}$, whereas each $k$-th column sum with $k \in\left\{1, \ldots,\left|E_{N^{\prime}}\right|\right\} \backslash\left\{\rho^{\sigma}(j) \mid j \in\{1, \ldots,|N|\}\right\}$ is equal to 0 .

Proof First note that by Remark 2 and the definition of obligation map the matrix $D^{\sigma, \hat{o}}$ is nonnegative.

The sum of the elements in each row $i \in N$ is equal to 1 because

$$
\sum_{k=1}^{\left|E_{N^{\prime}}\right|}\left(\hat{o}_{i}\left(\pi^{\sigma, k-1}\right)-\hat{o}_{i}\left(\pi^{\sigma, k}\right)\right)=\hat{o}_{i}\left(\pi^{\sigma, 0}\right)-\hat{o}_{i}\left(\pi^{\sigma,\left|E_{N^{\prime}}\right|}\right)=1-0=1
$$

for each $i \in N$.

The $\rho^{\sigma}(j)$-th column sums, for each $j \in\{1, \ldots,|N|\}$, are equal to 1 because

$$
\begin{aligned}
& \sum_{i \in N} D_{i \rho^{\sigma}(j)}^{\sigma, \hat{o}}=\sum_{i \in N}\left(\hat{o}_{i}\left(\pi^{\sigma, \rho^{\sigma}(j)-1}\right)-\hat{o}_{i}\left(\pi^{\sigma, \rho^{\sigma}(j)}\right)\right)= \\
& =\sum_{i \in N} \hat{o}_{i}\left(\pi^{\sigma, \rho^{\sigma}(j)-1}\right)-\sum_{i \in N} \hat{o}_{i}\left(\pi^{\sigma, \rho^{\sigma}(j)}\right)= \\
& =\left(\left|\pi^{\sigma, \rho^{\sigma}(j)-1}\right|-1\right)-\left(\left|\pi^{\sigma, \rho^{\sigma}(j)}\right|-1\right)=1
\end{aligned}
$$

for each $j \in\{1, \ldots,|N|\}$, where in the last equality we use Remark 2. The $k$-th column sums, for each $k \in\left\{1, \ldots,\left|E_{N^{\prime}}\right|\right\} \backslash\left\{\rho^{\sigma(j)} \mid j \in\{1, \ldots,|N|\}\right\}$, are equal to 0 because $\pi^{\sigma, k-1}=\pi^{\sigma, k}$ and then

$$
\sum_{i \in N} D_{i k}^{\sigma, \hat{o}}=\sum_{i \in N}\left(\hat{o}_{i}\left(\pi^{\sigma, k-1}\right)-\hat{o}_{i}\left(\pi^{\sigma, k}\right)\right)=0
$$

Definition 2 Let $\hat{o}$ be an obligation map on $\Theta(N \cup\{0\})$. Let $\sigma \in \Sigma_{E_{N^{\prime}}}$. We define the map $\phi^{\sigma, \hat{o}}: K^{\sigma} \rightarrow \mathbb{R}^{N}$ by

$$
\phi^{\sigma, \hat{o}}(w)=D^{\sigma, \hat{o}} w^{\sigma}
$$

for each mcst situation $w$ in the cone $K^{\sigma}$.

Onwards, let $e^{k} \in \mathbb{R}^{\left|E_{N^{\prime}}\right|}$ be the column vector such that $e_{i}^{k}=1$ if $i=k$ and $e_{i}^{k}=0$ for each $i \in\left\{1, \ldots,\left|E_{N^{\prime}}\right|\right\} \backslash\{k\}$. From Proposition 1 it follows directly that the matrix $\bar{D}^{\sigma, \hat{o}} \in \mathbb{R}^{N \times|N|}$ defined by

$$
\bar{D}^{\sigma, \hat{o}} e^{j}=D^{\sigma, \hat{o}} e^{\rho^{\sigma}(j)}
$$


for each $j \in N$ is a double stochastic matrix (i.e. all entries are nonnegative and each row sum and each column sum are equal to 1 ), and

$$
\phi^{\sigma, \hat{o}}(w)=\bar{D}^{\sigma, \hat{o}}\left(w\left(\sigma\left(\rho^{\sigma}(1)\right)\right), \ldots, w\left(\sigma\left(\rho^{\sigma}(|N|)\right)\right)\right)^{t} .
$$

In order to define Obligation rules properly on the set $\mathcal{W}^{N^{\prime}}$, we need Lemma 1. In the sequel, recall that, for each $t \in\left\{1, \ldots,\left|E_{N^{\prime}}\right|\right\}, w_{t}^{\sigma}$ is the $t$-th coordinate of the vector $w^{\sigma}$ as defined in the Preliminaries.

Lemma 1 Let ô be an obligation map on $\Theta(N \cup\{0\})$; let $\sigma \in \Sigma_{E_{N^{\prime}}}, w \in K^{\sigma}$. Suppose that, for some $t \in\left\{1, \ldots,\left|E_{N^{\prime}}\right|-1\right\}, w_{t}^{\sigma}=w_{t+1}^{\sigma}$. Then for the ordering $\sigma^{\prime} \in \Sigma_{E_{N^{\prime}}}$ such that $\sigma^{\prime}(i)=\sigma(i)$ for each $i \in\left\{1, \ldots,\left|E_{N^{\prime}}\right|\right\} \backslash$ $\{t, t+1\}, \sigma^{\prime}(t)=\sigma(t+1)$ and $\sigma^{\prime}(t+1)=\sigma(t)$, we have that $w \in K^{\sigma^{\prime}}$ and $\phi^{\sigma, \hat{o}}(w)=\phi^{\sigma^{\prime}, \hat{o}}(w)$.

Proof It is obvious that $w \in K^{\sigma^{\prime}}$. Let $a=w_{t}^{\sigma}$. Note that $\hat{o}\left(\pi^{\sigma, k}\right)=$ $\hat{o}\left(\pi^{\sigma^{\prime}, k}\right)$ for all $k \in\left\{1, \ldots,\left|E_{N^{\prime}}\right|\right\}$ with $k \neq t$. This implies that $w_{k}^{\sigma} D^{\sigma, \hat{o}} e^{k}=$ $w_{k}^{\sigma^{\prime}} D^{\sigma^{\prime}, \hat{o}} e^{k}$ for all $k \in\left\{1, \ldots,\left|E_{N^{\prime}}\right|\right\}$ with $k \notin\{t, t+1\}$ and

$$
\begin{aligned}
& w_{t}^{\sigma^{\prime}} D^{\sigma^{\prime}, \hat{o}} e^{t}+w_{t+1}^{\sigma^{\prime}} D^{\sigma^{\prime}, \hat{o}} e^{t+1}= \\
& =a\left(\hat{o}\left(\pi^{\sigma^{\prime}, t-1}\right)-\hat{o}\left(\pi^{\sigma^{\prime}, t}\right)\right)+a\left(\hat{o}\left(\pi^{\sigma^{\prime}, t}\right)-\hat{o}\left(\pi^{\sigma^{\prime}, t+1}\right)\right)= \\
& =a\left(\hat{o}\left(\pi^{\sigma^{\prime}, t-1}\right)-\hat{o}\left(\pi^{\sigma^{\prime}, t+1}\right)\right)=a\left(\hat{o}\left(\pi^{\sigma, t-1}\right)-\hat{o}\left(\pi^{\sigma, t+1}\right)\right)= \\
& =a\left(\hat{o}\left(\pi^{\sigma, t-1}\right)-\hat{o}\left(\pi^{\sigma, t}\right)\right)+a\left(\hat{o}\left(\pi^{\sigma, t}\right)-\hat{o}\left(\pi^{\sigma, t+1}\right)\right)= \\
& =w_{t}^{\sigma} D^{\sigma, \hat{o}} e^{t}+w_{t+1}^{\sigma} D^{\sigma, \hat{o}} e^{t+1} .
\end{aligned}
$$

So, $D^{\sigma, \hat{o}} w^{\sigma}=D^{\sigma^{\prime}, \hat{o}} w^{\sigma^{\prime}}$ or, equivalently, $\phi^{\sigma, \hat{o}}(w)=\phi^{\sigma^{\prime}, \hat{o}}(w)$.

By repeatedly using Lemma 5 we obtain

Proposition 2 Let $\hat{o}$ be an obligation map on $\Theta(N \cup\{0\})$. If $w \in K^{\sigma} \cap K^{\sigma^{\prime}}$ with $\sigma, \sigma^{\prime} \in \Sigma_{E_{N^{\prime}}}$, then $\phi^{\sigma, \hat{o}}(w)=\phi^{\sigma^{\prime}, \hat{o}}(w)$.

This proposition makes it possible to define an Obligation rule with respect to an obligation map on $\Theta(N \cup\{0\})$ as a map on $\mathcal{W}^{N^{\prime}}$.

Definition 3 Let $\hat{o}$ be an obligation map on $\Theta(N \cup\{0\})$. The Obligation (O-) rule w.r.t. $\hat{o}$ is the map $\phi^{\hat{o}}: \mathcal{W}^{N^{\prime}} \rightarrow \mathbb{R}^{N}$ defined by

$$
\phi^{\hat{o}}(w)=\phi^{\sigma, \hat{o}}(w)
$$

for each $w \in \mathcal{W}^{N^{\prime}}$, where $\sigma \in \Sigma_{E_{N^{\prime}}}$ is such that $w \in K^{\sigma}$.

Remark 3 The P-value (Branzei et al. (2003)) and the $P^{\tau}$-values, with $\tau \in$ $\Sigma_{N^{\prime}}$, introduced in Norde et al. (2004) and studied in Branzei et al.(2003), are Obligation rules. In fact $\phi^{\hat{o}^{*}}(w)=P(w)$ and $\phi^{\hat{o}^{\tau}}(w)=P^{\tau}(w)$ for each $\tau \in \Sigma_{N}$, where $\Sigma_{N}$ is the set of all bijections $\tau: N \rightarrow\{1, \ldots,|N|\}$. 
Now we make clear why we chose the name "Obligation rule". Let $\hat{o}$ an obligation map on $\Theta(N \cup\{0\})$ and let $w \in \mathcal{W}^{N^{\prime}}$. According to the corresponding O-rule $\phi^{\hat{o}}$, each player $i \in N$ has to pay fractions of edges summing up to 1 , which is the total obligation for player $i$ in the most situation $w$. Stated differently, an O-rule allocates the cost of an edge which forms in some step $k, k \in\left\{1, \ldots,\left|E_{N^{\prime}}\right|\right\}$, of the Kruskal algorithm to the players in $N$ according to the $k$-th column of the contribution matrix $D^{\sigma, \hat{o}}$, with $\sigma \in \Sigma_{E_{N^{\prime}}}$ such that $w \in K^{\sigma}$. After step $k$, by Proposition 1, the quantity of remaining obligations for each player $i \in N$ is given by $1-\sum_{j=1}^{k-1} D_{i j}^{\sigma, \hat{o}}=\hat{o}_{i}\left(\pi^{\sigma, k}\right)$.

We collect some interesting properties of O-rules in Proposition 3.

Proposition 3 The O-rules are efficient, satisfy the carrier property and form a convex set.

Proof Let $\hat{o}$ be an obligation map on $\Theta(N \cup\{0\})$, let $w \in \mathcal{W}^{N^{\prime}}$ and let $\sigma \in \Sigma_{E_{N^{\prime}}}$ be such that $w \in K^{\sigma}$.

i) From (3) and (6) it follows

$$
\phi_{i}^{\hat{o}}(w)=\sum_{k=1}^{|N|} \bar{D}_{i k}^{\sigma, \hat{o}} w\left(\sigma\left(\rho^{\sigma}(k)\right)\right)
$$

for each $i \in N$, implying that

$$
\sum_{i \in N} \phi_{i}^{\hat{o}}(w)=\sum_{k=1}^{|N|} w\left(\sigma\left(\rho^{\sigma}(k)\right)\right) \sum_{i \in N} \bar{D}_{i k}^{\sigma, \hat{o}}=\sum_{k=1}^{|N|} w\left(\sigma\left(\rho^{\sigma}(k)\right)\right)=w(\Gamma),
$$

where the second equality follows from Proposition 1 and where $\Gamma$ is a spanning network on $N^{\prime}$ of minimal cost. So efficiency is proved.

ii) Let $i \in N$ be a player who is $\left(w, N^{\prime}\right)$-connected to the source 0 . There exists $r \in\left\{1, \ldots,\left|E_{N^{\prime}}\right|\right\}$ such that $i$ is connected to 0 in $F^{\sigma, r}$ but not in $F^{\sigma, r-1}$ and $w(\sigma(r))=0$. Moreover, by the definition of an obligation map, $\hat{o}_{i}\left(\pi^{\sigma, k}\right)=0$ for $k \in\left\{r, \ldots,\left|E_{N^{\prime}}\right|\right\}$. It follows by $(6)$ that $\phi_{i}^{\hat{o}}(w)=$ 0 and then it is proved that $\phi^{\hat{o}}$ satisfies the carrier property.

iii) Let $\hat{o}^{\bullet}, \hat{o}^{\circ}$ and $\hat{o}^{\alpha}$, with $\alpha \in[0,1]$, be as in Remark 1. Then

$$
\begin{aligned}
& \alpha \phi^{\hat{o}^{\bullet}}(w)+(1-\alpha) \phi^{\hat{o}^{\circ}}(w)= \\
& =\alpha D^{\sigma, \hat{o}^{\bullet}} w^{\sigma}+(1-\alpha) D^{\sigma, \hat{o}^{\circ}} w^{\sigma}= \\
& =\left(\alpha D^{\sigma, \hat{o}^{\bullet}}+(1-\alpha) D^{\sigma, \hat{o}^{\circ}}\right) w^{\sigma}= \\
& =D^{\sigma, \hat{o}^{\alpha}} w^{\sigma}=\phi^{\hat{o}^{\alpha}}(w)
\end{aligned}
$$


for every $w \in \mathcal{W}^{N^{\prime}}$ and $\sigma \in \Sigma_{E_{N^{\prime}}}$ such that $w \in K^{\sigma}$, where the third equality follows from Remark 1 and the definition of $D^{\sigma, \hat{o}^{\alpha}}$. Then it is proved that the set of O-rules is a convex set.

We end this section with a proposition that enlightens the connection between the $P$-value and the $P^{\tau}$-values, $\tau \in \Sigma_{E_{N^{\prime}}}$, according to Remark 3 .

Proposition 4 Let $w \in \mathcal{W}^{N^{\prime}}$. Then

$$
P(w)=\frac{1}{n !} \sum_{\tau \in \Sigma_{N}} P^{\tau}(w) .
$$

Proof By Remark 3 and (4) we have only to prove that

$$
\bar{D}^{\sigma, \hat{o}^{*}}=\frac{1}{n !} \sum_{\tau \in \Sigma_{N}} \bar{D}^{\sigma, \hat{o}^{\tau}}
$$

Let $\sigma \in \Sigma_{E_{N^{\prime}}}$ be such that $w \in K^{\sigma}$.

To prove (8), note that for each $i \in\{1, \ldots,|N|\}$, the edge $\sigma\left(\rho^{\sigma}(i)\right)$ connects two disconnected subsets of vertices $S, T \in \pi^{\sigma, \rho^{\sigma}(i-1)}$. Then, for each player $j \in N \backslash(S \cup T)$, if any, $\frac{1}{n !} \sum_{\tau \in \Sigma_{N}} \bar{D}_{j i}^{\sigma \hat{o}^{\tau}}=\bar{D}_{j i}^{\sigma, \hat{o}^{*}}=0$.

On the other hand, for players in $S \cup T$, we have two possibilities regarding the position of the source w.r.t. the sets $S$ and $T$ :

i) The source 0 belongs neither to $S$ nor to $T$ implying that for each $j \in T$ and for each $\tau \in \Sigma_{N}$

$$
\hat{o}_{j}^{\tau}\left(\pi^{\sigma, \rho^{\sigma}(i-1)}\right)-\hat{o}_{j}^{\tau}\left(\pi^{\sigma, \rho^{\sigma}(i)}\right)= \begin{cases}1 & \text { if } \tau(j)=\min \{\tau(k) \mid k \in T\} \text { and } \\ & \tau(j) \neq \min \{\tau(k) \mid k \in S \cup T\} \\ 0 & \text { otherwise. }\end{cases}
$$

The fraction of orderings $\tau \in \Sigma_{N}$ such that $\arg \min \{\tau(k) \mid k \in S \cup T\} \in S$ is equal to $\frac{|S|}{|S \cup T|}=\frac{|S|}{|S|+|T|}$ whereas the fraction of such orderings $\tau \in \Sigma_{N}$ such that $\tau(j)=\min \{\tau(k) \mid k \in T\}$ is equal to $\frac{1}{|T|}$. Then it follows that for each $j \in T$

$$
\begin{aligned}
& \frac{1}{n !} \sum_{\tau \in \Sigma_{N}} \bar{D}_{j i}^{\sigma, \hat{o}^{\tau}}= \\
& =\frac{1}{n !} \sum_{\tau \in \Sigma_{N}}\left(\hat{o}_{j}^{\tau}\left(\pi^{\sigma, \rho^{\sigma}(i-1)}\right)-\hat{o}_{j}^{\tau}\left(\pi^{\sigma, \rho^{\sigma}(i)}\right)\right)= \\
& =\frac{|S|}{|S \cup T|} \frac{1}{|T|}=\frac{1}{|T|}-\frac{1}{|S \cup T|}= \\
& =\hat{o}_{j}^{*}\left(\pi^{\sigma, \rho^{\sigma}(i-1)}\right)-\hat{o}_{j}^{*}\left(\pi^{\sigma, \rho^{\sigma}(i)}\right)= \\
& =\bar{D}_{j i}^{\sigma, \hat{o}^{*}} .
\end{aligned}
$$


Similar arguments hold for each $j \in S$ too.

ii) The source 0 belongs either to $S$ or to $T$. Without loss of generality, suppose $0 \in S$. Then, for each $j \in S$

$$
\begin{aligned}
& \frac{1}{n !} \sum_{\tau \in \Sigma_{N}} \bar{D}_{j i}^{\sigma, \hat{o}^{\tau}}= \\
& =\frac{1}{n !} \sum_{\tau \in \Sigma_{N}}\left(\hat{o}_{j}^{\tau}\left(\pi^{\sigma, \rho^{\sigma}(i-1)}\right)-\hat{o}_{j}^{\tau}\left(\pi^{\sigma, \rho^{\sigma}(i)}\right)\right)= \\
& =0= \\
& =\hat{o}_{j}^{*}\left(\pi^{\sigma, \rho^{\sigma}(i-1)}\right)-\hat{o}_{j}^{*}\left(\pi^{\sigma, \rho^{\sigma}(i)}\right)= \\
& =\bar{D}_{j i}^{\sigma, \hat{o}^{*}} .
\end{aligned}
$$

On the other hand, for each $j \in T$

$$
\begin{aligned}
& \frac{1}{n !} \sum_{\tau \in \Sigma_{N}} \bar{D}_{j i}^{\sigma, \hat{o}^{\tau}}= \\
& =\frac{1}{n !} \sum_{\tau \in \Sigma_{N}}\left(\hat{o}_{j}^{\tau}\left(\pi^{\sigma, \rho^{\sigma}(i-1)}\right)-\hat{o}_{j}^{\tau}\left(\pi^{\sigma, \rho^{\sigma}(i)}\right)\right)= \\
& =\frac{1}{|T|}= \\
& =\hat{o}_{j}^{*}\left(\pi^{\sigma, \rho^{\sigma}(i-1)}\right)-\hat{o}_{j}^{*}\left(\pi^{\sigma, \rho^{\sigma}(i)}\right)= \\
& =\bar{D}_{j i}^{\sigma, \hat{o}^{*}} .
\end{aligned}
$$

A similar argument holds if $0 \in T$.

Hence (8) is proved and $P(w)=\frac{1}{n !} \sum_{\tau \in \Sigma_{N}} P^{\tau}(w)$.

An alternative proof of Proposition 4 is given in Branzei et al. (2004).

\section{Cost Monotonicity and PMAS}

In this section we will discuss some nice monotonicity properties of the Orules. First, we provide the definition of cost monotonic solutions for mcst situations.

Definition 4 A solution $F: \mathcal{W}^{N^{\prime}} \rightarrow \mathbb{R}^{N}$ is a cost monotonic solution if for all mcst situations $w, \bar{w} \in \mathcal{W}^{N^{\prime}}$ such that $w(\bar{e}) \leq \bar{w}(\bar{e})$ for one edge $\bar{e} \in E_{N^{\prime}}$ and $w(e)=\bar{w}(e)$ for each $e \in E_{N^{\prime}} \backslash\{\bar{e}\}$, it holds that $F(w) \leq F(\bar{w})$.

We prove in Theorem 1 that O-rules are cost monotonic; the main step is the following lemma.

Lemma 2 Let $\hat{o}$ be an obligation map on $\Theta(N \cup\{0\})$ and let $w \in \mathcal{W}^{N^{\prime}}$. Let $\bar{e} \in E_{N^{\prime}}$ and let $h>w(\bar{e})$ be such that there is no $e \in E_{N^{\prime}}$ with $w(\bar{e})<$ $w(e)<h$. Define $\tilde{w} \in \mathcal{W}^{N^{\prime}}$ by $\tilde{w}(e):=w(e)$ if $e \in E_{N^{\prime}} \backslash\{\bar{e}\}$ and $\tilde{w}(\bar{e})=h$. Then: $\phi^{\hat{o}}(\bar{w}) \geq \phi^{\hat{o}}(w)$. 
Proof Let $K:=\left\{e \in E_{N^{\prime}} \mid w(e)=w(\bar{e})\right\}$ be the set of edges that have the same cost as $\bar{e}$. Let $\sigma \in \Sigma_{E_{N^{\prime}}}$ be such that $w \in K^{\sigma}$. Without loss of generality we may assume that $\sigma^{-1}(\bar{e})=\max \left\{\sigma^{-1}(e) \mid e \in K\right\}$, i.e. $\sigma$ ranks the edges of $K$ with $\bar{e}$ last. By construction we also have $\tilde{w} \in K^{\sigma}$ and hence

$$
\phi^{\hat{o}}(\tilde{w})=D^{\sigma, \hat{o}} \tilde{w}^{\sigma} \geq D^{\sigma, \hat{o}} w^{\sigma}=\phi^{\hat{o}}(w),
$$

where at the inequality we used the fact that $\tilde{w}^{\sigma} \geq w^{\sigma}$ and the fact that the matrix $D^{\sigma, \hat{o}}$ is nonnegative.

Theorem 1 Obligation rules are cost monotonic.

Proof Let $\hat{o}$ be an obligation map on $\Theta(N \cup\{0\})$ and let $\phi^{\hat{o}}$ the O-rule w.r.t $\hat{o}$. Let $w, \bar{w} \in \mathcal{W}^{N^{\prime}}$ be as in Definition 4 .

Let $H:=\left\{h \in \mathbb{R} \mid\right.$ there is an edge $f \in E_{N^{\prime}}$ s.t. $\left.h=w(f) \in(w(e), \bar{w}(\bar{e}))\right\}$. If $H=\emptyset$ then the statement follows directly from Lemma 2. If $H \neq \emptyset$ write $H=\left\{h_{1}, \ldots, h_{k}\right\}$ with $h_{1}<\ldots<h_{k}$.

Consider the sequence of precisely $k+2$ mcst situations $w_{0}, \ldots, w_{k+1} \in$ $\mathcal{W}^{N^{\prime}}$ such that $w_{0}=w, w_{k+1}=\bar{w}$ and for each $r \in\{1, \ldots, k\}, w_{r}(e)=w(e)$ for each $e \in E_{N^{\prime}} \backslash\{\bar{e}\}$ and $w_{r}(\bar{e})=h_{r}$.

Applying Lemma 2 for each $r \in\{1, \ldots,|H|\}$, with $w_{r-1}$ in the role of $w$ and $w_{r}$ in the role of $\tilde{w}$, it follows that

$$
\phi^{\hat{o}}(\bar{w})=\phi^{\hat{o}}\left(w_{k+1}\right) \geq \phi^{\hat{o}}\left(w_{k}\right) \ldots \geq \phi^{\hat{o}}\left(w_{0}\right)=\phi^{\hat{o}}(w),
$$

which finally proves cost monotonicity of O-rules.

By Theorem 1 and Remark 3 the $P$-value and the $P^{\tau}$-values, for each $\tau \in \Sigma_{N}$, are cost monotonic O-rules. The following example illustrates the cost monotonicity of the $P$-value.

Example 6 Consider the mcst situation $\left\langle N^{\prime}, w>\right.$ with $N^{\prime}=\{0,1,2,3\}$ and $w$ as depicted in Figure 1. The contribution matrix $D^{\sigma, \hat{o}^{*}}$ is

$$
D^{\sigma, \hat{o}^{*}}=\left(\begin{array}{cccccc}
\frac{1}{2} & \frac{1}{6} & 0 & \frac{1}{3} & 0 & 0 \\
0 & \frac{2}{3} & 0 & \frac{1}{3} & 0 & 0 \\
\frac{1}{2} & \frac{1}{6} & 0 & \frac{1}{3} & 0 & 0
\end{array}\right)
$$

and $w^{\sigma}=(10,18,20,24,24,26)^{t}$.

Then $P(w)=\phi^{\hat{o}^{*}}(w)=D^{\sigma, \hat{o}^{*}} w^{\sigma}=(16,20,16)^{t}$. 
Now we consider the mcst situation $\left\langle N^{\prime}, \bar{w}>\in \mathcal{W}^{N^{\prime}}\right.$ where $\bar{w}$ is equal to $w$ except for edge $\{1,3\}$ whose cost is now $\bar{w}(\{1,3\})=26$. The contribution matrix for $\bar{w}$ is

$$
D^{\bar{\sigma}, \hat{o}^{*}}=\left(\begin{array}{cccccc}
\frac{1}{2} & \frac{1}{6} & \frac{1}{3} & 0 & 0 & 0 \\
\frac{1}{2} & \frac{1}{6} & \frac{1}{3} & 0 & 0 & 0 \\
0 & \frac{2}{3} & \frac{1}{3} & 0 & 0 & 0
\end{array}\right)
$$

and $\bar{w}^{\sigma}=(18,20,24,24,26,26)^{t}$. Therefore

$$
\begin{gathered}
\phi^{\hat{o}^{*}}(w)=P(w)=\left(\begin{array}{cccccc}
\frac{1}{2} & \frac{1}{6} & \frac{1}{3} & 0 & 0 & 0 \\
\frac{1}{2} & \frac{1}{6} & \frac{1}{3} & 0 & 0 & 0 \\
0 & \frac{2}{3} & \frac{1}{3} & 0 & 0 & 0
\end{array}\right)\left(\begin{array}{c}
18 \\
20 \\
24 \\
24 \\
26 \\
26
\end{array}\right)= \\
=\left(9+\frac{10}{3}+8,9+\frac{10}{3}+8, \frac{40}{3}+8\right)^{t}=\left(\frac{61}{3}, \frac{61}{3}, \frac{64}{3}\right)^{t} .
\end{gathered}
$$

The following theorem shows that O-rules induce a pmas for the corresponding most games.

Before introducing the theorem, we need to introduce some further notations. Let $o$ be an obligation function and $\hat{o}$ the corresponding obligation map. Let $S \subseteq N$, let $o_{S}$ denote the restriction of $o$ to $2^{S} \backslash\{\emptyset\}$ and let $\hat{o}_{S}$ denote the corresponding obligation map, i.e.

$$
\hat{o}_{S}(\theta)=\sum_{T \in \theta, 0 \notin T} o_{S}(T)
$$

for every $\theta \in \Theta(S \cup\{0\})$.

Recall also that if $w \in \mathcal{W}^{N^{\prime}}$, then an O-rule $\phi^{\hat{o}_{S}}$ w.r.t the obligation map $\hat{o}_{S}$ and applied to $w_{\mid S^{\prime}}$, i.e. the restriction of the weight function $w$ to $E_{S^{\prime}} \subseteq E_{N^{\prime}}$ as defined in the Preliminaries, provides a vector in $\mathbb{R}^{S}$ according to Definition 3 w.r.t. the set of nodes $S^{\prime}$.

Theorem 2 Let $\hat{o}$ be an obligation map on $\Theta(N \cup\{0\})$, let $\phi^{\hat{o}}$ the O-rule w.r.t $\hat{o}$, and let $w \in \mathcal{W}^{N^{\prime}}$. Then the table $\left[\phi^{\hat{o}_{S}}\left(w_{\mid S^{\prime}}\right)\right]_{S \in 2^{N} \backslash\{\emptyset\}}$ is a pmas for the mcst game $\left(N, c_{w}\right)$.

Proof Given $S \subset T \subseteq N$, define $<T^{\prime}, \bar{w}>$ with $T^{\prime}=T \cup\{0\}$ and

$$
\bar{w}(\{i, j\})= \begin{cases}w(\{i, j\}) & \text { if } i, j \in S^{\prime} \\ w(\{i, j\})+\lambda_{S} & \text { otherwise }\end{cases}
$$


where $\lambda_{S}=1+\max \left\{w(\{i, j\}) \mid i, j \in S^{\prime}\right\}$.

Then, in $\left\langle T^{\prime}, \bar{w}\right\rangle$ each edge with at least one node not in $S^{\prime}$ is more expensive than in $<T^{\prime}, w_{\mid T^{\prime}}>$.

Further, let $\bar{\sigma} \in \Sigma_{E_{T^{\prime}}}$ be such that $\bar{w} \in K^{\bar{\sigma}}$ and let $\sigma^{S^{\prime}} \in \Sigma_{E_{S^{\prime}}}$ be such that $\sigma^{S^{\prime}}(i)=\bar{\sigma}(i)$ for each $i \in\left\{1, \ldots,\left|E_{S^{\prime}}\right|\right\}$. Then by (9) it follows that $w_{\mid S^{\prime}} \in K^{\sigma^{S^{\prime}}}$.

Note that for each $i \in S$

$$
\phi_{i}^{\hat{o}_{T}}(\bar{w})=\phi_{i}^{\hat{o}_{S}}\left(w_{\mid S^{\prime}}\right) .
$$

This follows from the fact that in $\left\langle S^{\prime}, w_{\mid S^{\prime}}\right\rangle$ the edges with at least one node not in $S^{\prime}$ are discarded and in $\left\langle T^{\prime}, \bar{w}>\right.$ the edges with at least one node not in $S^{\prime}$ are allowed but they are too expensive. The result is that applying the Kruskal procedure on $\left\langle T^{\prime}, \bar{w}>\right.$ w.r.t. $\bar{\sigma}$ the players in $S^{\prime}$ are already connected to 0 before one of the edges with nodes not in $S^{\prime}$ is considered. So, by definition of an obligation map, we have that the contribution matrix with $|T|$ rows and $\left|E_{T^{\prime}}\right|$ columns $D^{\bar{\sigma}, \hat{o}_{T}}$ is of the form

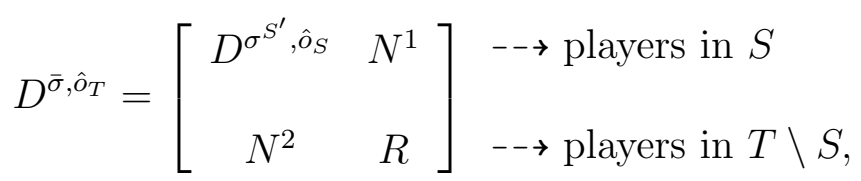

where the four submatrices $D^{\sigma^{S^{\prime}}{ }^{,}{ }_{S}}, N^{1}, N^{2}$ and $R$ are such that:

- $D^{\sigma^{S^{\prime}}{ }_{\hat{o}_{S}}}$ is the contribution matrix w.r.t. to $\sigma^{S^{\prime}}$ and to $\hat{o}_{S}$ with $|S|$ rows and $\left|E_{S^{\prime}}\right|$ columns;

- $N^{1}$ is the null matrix with $|S|$ rows and $\left|E_{T^{\prime}}\right|-\left|E_{S^{\prime}}\right|$ columns;

- $N^{2}$ is the null matrix with $|T|-|S|$ rows and $\left|E_{S^{\prime}}\right|$ columns;

- $R$ is a real valued matrix with $|T|-|S|$ rows and $\left|E_{T^{\prime}}\right|-\left|E_{S^{\prime}}\right|$ columns obtained according to the definition of the contribution matrix $D^{\bar{\sigma}, \hat{o}_{T}}$.

Hence, for each $i \in S, \phi_{i}^{\hat{o}_{T}}(\bar{w})=\left(D^{\sigma^{S^{\prime}}{ }^{\hat{o}_{S}}} \bar{w}_{\mid S^{\prime}}^{\sigma^{S^{\prime}}}\right)_{i}=\phi_{i}^{\hat{o}_{S}}\left(\bar{w}_{\mid S^{\prime}}\right)=\phi_{i}^{\hat{o}_{S}}\left(w_{\mid S^{\prime}}\right)$, which yields equation $(10)$. [Here $\left(D^{\sigma^{S^{\prime}}, \hat{o}_{S}} \bar{w}_{\mid S^{\prime}}^{\sigma^{S}}\right)_{i}$ is the $i$-th component of the vector $D^{\sigma^{S^{\prime}}, \hat{o}_{S}} \bar{w}_{\mid S^{\prime}}^{\sigma^{S^{\prime}}}$.]

Recall that O-rules are cost monotonic. Since $\bar{w}(e) \geq w_{\mid T^{\prime}}(e)$ for each $e \in E_{T^{\prime}}$, then

$$
\phi_{i}^{\hat{o}_{T}}(\bar{w}) \geq \phi_{i}^{\hat{o}_{T}}\left(w_{\mid T^{\prime}}\right), \quad \text { for each } i \in T \text {. }
$$


From (10) and (11) we obtain

$$
\phi_{i}^{\hat{o}_{S}}\left(w_{\mid S^{\prime}}\right) \geq \phi_{i}^{\hat{o}_{T}}\left(w_{\mid T^{\prime}}\right) \quad \text { for each } i \in S
$$

From (12) and the efficiency property it follows that $\left[\phi^{\hat{o}_{S}}\left(w_{\mid S^{\prime}}\right)\right]_{S \in 2^{N} \backslash\{\emptyset\}}$ is a pmas for the mcst game $\left(N, c_{w}\right)$

From Theorem 2 and the definition of a pmas, it follows that O-rules provide cost allocations which are core elements of the game $\left(N, c_{w}\right)$.

Example 7 Consider again the mcst situation $<N^{\prime}, w>$ with $N^{\prime}=\{0,1,2$, $3\}$ and $w$ as depicted in Figure 1. Then the P-value, as the O-rule $\phi^{\hat{o}^{*}}(w)$ previously introduced, applied to each mcst situation $<S \cup\{0\}, w_{\mid S \cup\{0\}}>$, provides the following population monotonic allocation scheme

$$
\left[\phi_{i}^{\hat{o}_{S}^{*}}\left(w_{\mid S^{\prime}}\right)\right]_{S \in 2^{N} \backslash\{\emptyset\}, i \in S}=\left[P_{i}\left(w_{\mid S^{\prime}}\right)\right]_{S \in 2^{N} \backslash\{\emptyset\}, i \in S}=\left\{\begin{array}{c|c||c|c|c|}
\hline S & 1 & 2 & 3 \\
\hline 123 & 16 & 20 & 16 \\
12 & 21 & 21 & * \\
13 & 17 & * & 17 \\
23 & * & 22 & 22 \\
1 & 24 & * & * \\
2 & * & 24 & * \\
3 & * & * & 26 \\
\hline
\end{array}\right.
$$

\section{Final remarks}

This paper considers the class of Obligation rules and studies their monotonicity properties. They cover old results in Branzei et al. (2003) and Norde et al. (2004). In the former, an axiomatic characterization of the $P$-value for mcst situations is given. In the latter, existence of a pmas for mcst games is proved. In this paper we introduce a class of solutions for mcst situations, the Obligation rules, which are cost monotonic, induce a pmas and, as already said in Remark 3, include among others the $P$-value and the $P^{\tau}$-values, for each $\tau \in \Sigma_{N}$.

Further, it turns out that the class of O-rules is a subclass of the Construct and Charge rules introduced and studied in Moretti et al. (2004), which are also defined via a matrix product with the unique difference that the columns in the contribution matrix do not necessarily derive from obligation maps.

Of course, other rules which are not of Obligation type can be cost monotonic rules. For instance, the egalitarian rule, which allocates to each player $i \in N$ an equal amount of the total cost of the mest, is cost monotonic but 
it is not an O-rule, since it does not satisfy the carrier property. Moreover, the cost allocation provided by the egalitarian rule is generically not a core element implying that this rule does not induce any pmas.

In Proposition 4, we illustrate a strong connection between the $P$-value and the $P^{\tau}$-values, for each $\tau \in \Sigma_{N}$. In Branzei et al. (2004) we characterize the link between these solutions, based on the notion of irreducible core. Roughly speaking, given a mcst situation $w \in \mathcal{W}^{N^{\prime}}$, the irreducible core of the mcst game corresponding to $w$ is the core of the concave mcst game corresponding to a mest situation which is obtained via an adaptation of $w$ introduced in Bird (1976). In Branzei et al. (2004), it is proved that the $P^{\tau}$-values, with $\tau \in \Sigma_{N}$, are extreme points of the irreducible core and that the $P$-value coincides with the Shapley value of the concave mcst game corresponding to the adaptation of $w$. This last fact is proven in an alternative way in Bergantinos and Vidal-Puga (2004b).

For axiomatic characterizations of the $P$-value see Feltkamp et al. (1994), Branzei et al. (2003), Bergantiños and Vidal-Puga (2004a).

\section{References}

Aarts, H. (1994), Minimum cost spanning tree games and set games, PhD Dissertation, University of Twente, The Netherlands.

Bird, C.G. (1976), On cost allocation for a spanning tree: a game theoretic approach, Networks, 6, 335-350.

Bergañtinos, G., Vidal-Puga, J.J. (2004a), Additivity in cost spanning tree problems, EconWPA, RePEc:wpa:wuwpga: 0405001

Bergañtinos, G., Vidal-Puga, J.J. (2004b), Defining rules in cost spanning tree problems through the canonical form, EconPapers, RePEc:wpa:wuwpga: 0402004.

Branzei, R., Moretti, S., Norde, H., Tijs, S., (2003), The $P$-value for cost sharing in minimum cost spanning tree situations, CentER DP 2003-129, Tilburg University, The Netherlands (to appear in Theory and Decision, Kluwer ed.).

Branzei, R., Moretti, S., Norde, H., Tijs, S., (2004), Cost monotonic rules and the irreducible core for connection problems, Working paper. 
Dutta, B., Kar, A. (2002), Cost monotonicity, consistency and minimum cost spanning tree games, University of Warwick, Mimeo.

Feltkamp, V. (1995), Cooperation in controlled network structures, PhD Dissertation, Tilburg University, The Netherlands.

Feltkamp, V., Tijs, S., Muto, S. (1994), On the irreducible core and the equal remaining obligations rule of minimum cost spanning extension problems, CentER DP 106, Tilburg University, The Netherlands.

Granot, D., Huberman, G. (1981), On minimum cost spanning tree games, Mathematical Programming, 21, 1-18.

Kent, K.J., Skorin-Kapov, D. (1996), Population monotonic cost allocations on MSTs, DP, State University of New York at Stony Brook.

Kruskal, J.B. (1956), On the shortest spanning subtree of a graph and the traveling salesman problem, Proceedings of the American Mathematical Society, $7,48-50$.

Moretti, S., Norde, H., Pham Do, K.H., Tijs, S. (2002), Connection problems in mountains and monotonic allocation schemes, Top, 10, 83-99.

Moretti, S., Tijs, S., Branzei, R., Norde, H., (2004), Cost monotonic 'construct and charge' rules, Working Paper.

Norde, H., Moretti, S., Tijs, S. (2004), Minimum cost spanning tree games and population monotonic allocation schemes, European Journal of Operational Research, 154, 84-97.

Sprumont, Y. (1990), Population monotonic allocation schemes for cooperative games with transferable utility, Games and Economic Behavior, 2, 378-394. 